\title{
Magnetic Exchange Interactions in Binuclear and Tetranuclear Iron (III) Complexes Described by Spin-Flip DFT and Heisenberg Effective Hamiltonians
}

\author{
Saikiran Kotaru, Maristella Alessio and Anna I. Krylov ${ }^{a}$ \\ Department of Chemistry, University of Southern California, Los Angeles, California 90089-0482 \\ a Corresponding author: krylov@usc.edu
}

Low-energy spectra of single-molecule magnets (SMMs) are often described by the Heisenberg Hamiltonian. Within this formalism, exchange interactions between magnetic centers determine the ground-state multiplicity and energy separation between the ground and excited states. In this contribution, we extract exchange coupling constants $(J)$ for a set of iron (III) binuclear and tetranuclear complexes from allelectron calculations using non-collinear spin-flip time-dependent density functional theory (NC-SF-TDDFT). For the series of binuclear complexes with $J$-values ranging from -6 to $-132 \mathrm{~cm}^{-1}$, our benchmark calculations using the short-range hybrid LRC- $\omega$ PBEh functional and 6-31G(d,p) basis set agree well (mean absolute error of $4.7 \mathrm{~cm}^{-1}$ ) with the experimentally derived values. For the tetranuclear SMMs, the computed $J$ constants are within $6 \mathrm{~cm}^{-1}$ from the values extracted from the experiment. We explore the range of applicability of the Heisenberg model by analyzing the radical character in the binuclear iron (III) complexes using natural orbitals (NO) and their occupations. On the basis of the number of effectively unpaired electrons and the NO occupancies, we attribute larger errors observed in strongly anti-ferromagnetic species to an increased ionic character. The results illustrate the efficiency of the spin-flip protocol for computing the exchange couplings and the utility of the NO analysis in assessing the validity of effective spin Hamiltonians.

\section{INTRODUCTION}

Single-molecule magnets (SMMs) are of interest due to their potential use in high-density information storage, spintronics, and quantum computing[1-5]. Rational tuning of their ground-state spin, magnetic anisotropy, and magnetic exchange couplings is essential in the 
design of SMMs with desired magnetic behavior (i.e., ferromagnetic or antiferromagnetic, switchable, etc.)[6]. For example, magnetic properties of SMMs arise due to their spinordering, which in turn is governed by the exchange interactions between the localized effective spins. In addition, the magnitude of the exchange coupling controls the magnetic relaxation rate, as high exchange results in higher energy separations between the ground and excited states, thereby suppressing faster magnetic relaxation via close-lying spin states [7, 8]. High-spin transition-metal compounds including multiple magnetic centers, such as iron (III), iron (II), and manganese (III), are well suited for the design of SMMs[9-12]. Among the best examples, a cluster of four iron (III) spins with $S=5$ spin ground state shows magnetic bistability for times as long as hundreds of nanoseconds, and, consequently, was proposed as a qubit candidate (i.e., two-level system) for the implementation of moleculebased quantum computers[9]. Similar magnetic frameworks anchored to a conducting surface were also investigated in the context of molecular spintronics[13]. Despite the observation of magnetic hysteresis in such multi-nuclear complexes, their low blocking temperature prevents their incorporation into a real quantum device, and calls for a joint effort by experiment and theory to tweak the SMMs magnetic properties through optimization of their chemical structures.

First-principles calculations of exchange interactions in transition-metal complexes are challenging because small energy gaps between the spin states of interest require accurate treatment of both static and dynamic correlation effects. Traditionally, such systems have been described by multi-reference methods, such as complete active space self-consistent field methods augmented with perturbative corrections. For example, CASPT2[14] and NEVPT2[15] (n-electron valence-state PT) are frequently used in studies of magnetic molecules, providing accurate estimates of their magnetic properties[16, 17]. Due to the high cost of such multi-reference calculations, more affordable DFT-based approaches are often employed, such as the broken symmetry (BS) methods[18-20]. Notwithstanding its wide use, the limitations of BS-DFT are well known - it is based on unphysical brokensymmetry solutions and its application relies on somewhat arbitrary choice of projectors. Furthermore, BS-DFT does not scale well with the number of radical sites, as the number of BS solutions grows rapidly.

We follow an alternative strategy based on the spin-flip approach. Spin-flip (SF) methods[21-27] offer a balanced treatment of multi-configurational states of polyradicals 
within a single-reference formalism, making the SF-protocol suitable for applications to SMMs[28, 29]. The SF approach does not rely on scrambled spin-states and does not require choosing an active space. When combined with DFT[24, 25], the spin-flip approach provides a computationally more efficient yet reliable approach to tackle extended SMMs.

Following previous SF studies of SMMs (see Ref. [28] and references therein), here we use non-collinear spin-flip time-dependent density functional theory (NC-SF-TDDFT) [25, 30] to calculate exchange interactions in binuclear and tetranuclear Fe (III) complexes. In these molecules, each Fe (III) center has a $d^{5}$ electronic configuration, with local ground-state spin configuration ranging from hextet, quartet, and doublet states depending on the strength of the ligand field, as illustrated in Fig. 1.
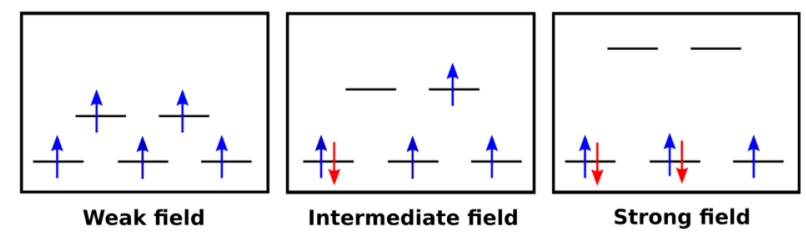

FIG. 1: Effect of the ligand-field strength on the local ground-state spin configuration of Fe (III) ions. The orbital splitting pattern corresponds to octahedral coordination. In the weak-field limit, the splitting between the $d$-orbitals is small and the low-lying manifold of electronic states is derived from configurations with five unpaired electrons, i.e., when each $d$-orbital is singly occupied, as prescribed by the Hund rule. In the opposite limit of strong field, the energy separation between the two orbital sets is larger than the electron pairing energy, giving rise to the low-spin configuration.

Magnetic properties of SMMs can be modeled using phenomenological spin Hamiltonians of varying complexity. These spin Hamiltonians contain parameters that can be determined either by fitting to experimental measurements or by using ab initio calculations[31]. Thus, spin Hamiltonians establish a connection between theory and experiment. The HeisenbergDirac-Van Vleck (HDvV) Hamiltonian is often employed to describe the inter-site effective exchange interactions. This model is expected to be suitable for treating exchange interactions in multi-center Fe (III) SMMs within the weak-field limit, when the non-Hund configurations (such as those in the middle and right panels of Fig. 1) are well separated in energy from the configurations in which the local electronic configuration has five unpaired electrons. We begin by assessing the applicability of the HDvV treatment in the binuclear complexes by employing density-based analysis and natural orbitals (NOs) $[28,32]$. We then investigate the functional and basis-set dependence on exchange coupling constants in binu- 
clear complexes[33-35]. For tetranuclear SMMs, we simplify the problem of parameterizing the Heisenberg Hamiltonian for a multi-center molecular magnet by performing only a single SF calculation from the highest-multiplicity state, following the approach of Mayhall and Head-Gordon[36].

The paper is organized as follows. Section II presents the spin-flip formalism and the theory of the HDvV Hamiltonians. Section III provides the computational details. Section IV A presents the natural orbital analysis of electronic states in mono- and bi- nuclear complexes, followed by benchmark results for binuclear complexes in Section IV B and the calculations on tetranuclear SMMs in Section IV C. Our concluding remarks are given in Section V.

\section{THEORETICAL FRAMEWORK}

To describe spin states of the Fe(III) complexes under study, we rely on the spin-flip (SF) method. In the SF methods[21, 22, 24, 37], high-spin states are used as a reference from which all the multi-configurational lower-spin states can be obtained by spin-flipping excitations. The high-spin state does not exhibit strong correlation and thus its wave function can be accurately described by any single-reference method, including standard Kohn-Sham DFT, and then used as a reference to access the lower-spin manifold as "excited" states in the spin-flipped sector of the Fock space. Within the SF approach, the target low-spin states are described as

$$
\Psi_{M_{s}=S-1}^{S, S-1}=\hat{R}_{M_{s}=-1} \Psi_{M_{s}=S}^{S}
$$

where the spin-flip operator $\hat{R}_{M_{s}=-1}$ generates all possible singly excited determinants in which the spin of one electron is flipped with respect to the high-spin reference (i.e., KohnSham reference in the SF-TDDFT formulation, see Refs. [24, 37]). SF methods have shown robust performance in treating diradicals[25, 38], triradicals[39, 39, 40, 40-42], conical intersections[43-47], and metal-containing SMMs[28, 29, 33].

Phenomenological spin Hamiltonians are commonly used to interpret magnetic measurements and serve as a bridge between theory and experiment[31, 48]. The low-energy spec- 
trum of a system of interacting spins can be described by the HDvV Hamiltonian[49-51],

$$
\hat{H}^{H D v V}=-\sum_{A, B} J_{A B} \hat{\mathbf{S}}_{A} \hat{\mathbf{S}}_{B},
$$

where operators $\hat{\mathbf{S}}_{A, B}$ are understood as effective local spins on centers $A$ and $B$, and $J_{A B}$ is the exchange coupling between them. The sign of $J_{A B}$ determines the nature of the exchange interactions: ferromagnetic $\left(J_{A B}>0\right)$ or antiferromagnetic $\left(J_{A B}<0\right)$.

Experimentally, the coupling constants are obtained by fitting temperature-dependent magnetic susceptibility to the $\mathrm{HDvV}$ model[52]. In contrast, a full-electron quantumchemistry calculation can, in principle, provide all electronic states of the system, and by analyzing the resulting energies and wave functions, one can then assess the validity of the spin Hamiltonian and determine the exchange interactions between the spin centers [31, 48]. However, such brute-force calculations of the entire manifold of low-lying states entail an exponential computational cost. This problem can be circumvented by an elegant approach presented by Mayhall and Head-Gordon[36], who demonstrated that one can construct and parameterize the HDvV Hamiltonian of Eq. (2) for an arbitrary number of unpaired electrons and metallic centers from the two highest-multiplicity states, the high-spin $|S\rangle$ and adjacent $|S-1\rangle$ spin states of $M_{s}=S-1$ components. These states can be computed by any spin-flip method (with a single spin-flip) from the highest-multiplicity reference state. Further details about Mayhall's approach can be found in Refs. $[36,53]$ and in the Supplementary Information (SI).

In binuclear systems, the procedure of extracting $J$-values is simplified by employing the Landé interval rule[54]:

$$
E(S)-E(S-1)=-2 S J_{A B},
$$

which is derived by solving the $\mathrm{HDvV}$ Hamiltonian analytically. Hence, within the $\mathrm{HDvV}$ model, a single spin-flip calculation is sufficient to compute the $J$-coupling between the two metallic centers. Mayhall and Head-Gordon validated this approach by comparing the single SF results against the full SF calculations (i.e., with multiple spin-flips) that directly compute all components of the multiplet[53]. In the case of multinuclear SMMs, the procedure of parameterizing the $\mathrm{HDvV}$ Hamiltonian requires additional post-processing 
steps and is based on the effective Hamiltonian theory as formalized by Bloch and described in details elsewhere[31, 36, 55]. This approach can provide a large number of exchange interaction couplings, enabling the validation of the $\mathrm{HDvV}$ model upon comparison with the effective Hamiltonian generated from the energies and wave functions of an all-electron $a b$ initio calculation.

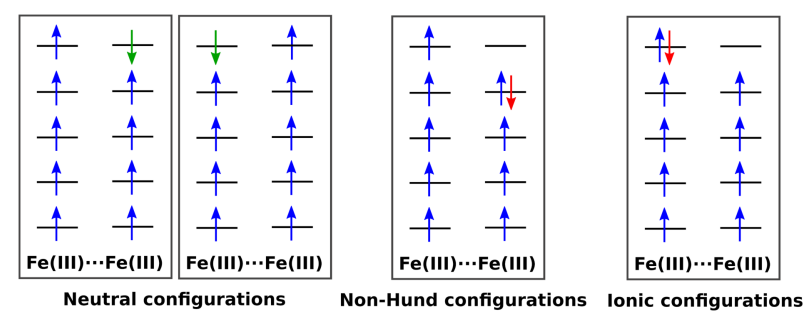

FIG. 2: Three types of configurations in Fe (III) binuclear SMMs: Neutral Hund-type (with fixed orbital occupations), locally excited (non-Hund-type with paired electrons), and ionic (in which the number of electrons on each center is different).

The bilinear exchange interactions of the HDvV model of Eq. (2) can be derived from the more-general Hubbard model using second-order quasidegenerate perturbation theory[31]. The derivation assumes a large energy gap between the manifold of states with fixed orbital occupations, such as all possible determinants with five unpaired electrons for Fe (III), and states with doubly occupied orbitals, such as locally excited non-Hund and ionic configurations in which the number of magnetic electrons on each center changes. Different types of configurations are shown in Fig. 2. When present with large weights, these non-Hund neutral and ionic configurations result in a non-Heisenberg spectral pattern, leading to discrepancies between theoretically and experimentally obtained $J$-couplings. In such cases, the HDvV model breaks down and models incorporating non-Hund configurations explicitly should be considered instead.

To analyze the deviations from the Heisenberg behavior, we employ natural orbitals and their occupations. Natural orbitals, which are eigenfunctions of the one-particle state density matrix[56-59], offer a compact description of the correlated wave functions. The corresponding eigenvalues can be interpreted as orbital occupations and used to quantify the radical character in a given electronic state. Following our previous work[28, 32], we use 
Head-Gordon's index[60] to compute the number of effectively unpaired electrons,

$$
n_{u, n l}=\sum_{i} \bar{n}_{i}^{2}\left(2-\bar{n}_{i}\right)^{2}
$$

where $\bar{n}_{i}$ is spin-averaged occupation of natural orbital $i$ and the sum runs over all natural orbitals. For the HDvV model to be valid in binuclear Fe (III) complexes, the low-lying spin states should have $n_{u, n l}=10\left(n_{u, n l}=20\right.$ for the tetranuclear ones $)$ and deviations from this value would indicate pairing of the active electrons. Similarly, the spin-traced occupancies of 10 frontier natural orbitals should be close to 1 . The appearance of locally excited non-Hund and ionic configurations would manifest itself in $n_{u, n l}<10$ and spin-traced occupancies of the frontier orbitals different from 1.

\section{COMPUTATIONAL DETAILS}

To asses the performance of the HDvV model, we extract $J$-couplings for a set of antiferromagnetic Fe (III) SMMs (Fig. 3)[61-76]. Below we refer to them by the numbers as shown in Fig. 3. Several of these complexes have been studied using broken symmetry DFT (BS-DFT) approach by Joshi et al.[35]. All these complexes have Fe (III) atoms with $d^{5}$ configuration. In binuclear complexes 1-12, the Fe atoms are connected by a dibridged oxo unit (complexes 1-4), single oxo unit (complexes 5-7), oxo and acetate bridges (complexes 8-12). In the tetranuclear complexes 13 and 14, the Fe atoms are bridged by oxymethyl groups whereas 15 and 16 have both oxo and acetate bridges. As shown by the experimental studies and confirmed by our calculations, the kind of linkage between Fe atoms plays a key role in determining the exchange coupling.

In addition to these multi-nuclear Fe (III) compounds, we also examined three model mononuclear Fe (III) systems of varying crystal-field strengths: $\left[\mathrm{Fe}(\mathrm{Cl})_{6}\right]^{3-}$ (weak field), a monomer unit built from ABIZOA complex (ABI-m), and $\left[\mathrm{Fe}(\mathrm{CN})_{6}\right]^{3-}$ (strong field). The ABI-m complex consists of benzimidazole, azide, and ethanolamine ligands. For $\left[\mathrm{Fe}(\mathrm{Cl})_{6}\right]^{3-}$

and $\left[\mathrm{Fe}(\mathrm{CN})_{6}\right]^{3-}$, we used PCM solvent to stabilize the multiply charged anions. Corresponding structures are given in the SI.

All calculations were carried out using the Q-Chem electronic structure package[77, 78]. We used high-spin reference states of $S=5$ and $S=10$ to compute spin-flip states in the 




Complex 1: ELISAU

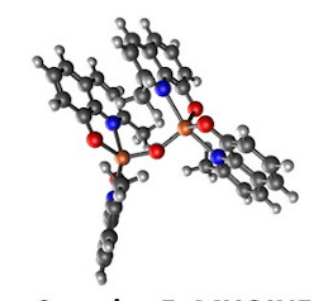

Complex 5: MHQINF

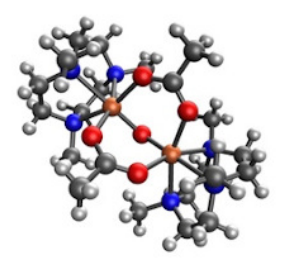

Complex 9: DIBXAN10



Complex 13: AGAQIJ

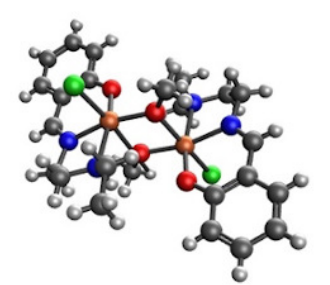

Complex 2: BAZCOV

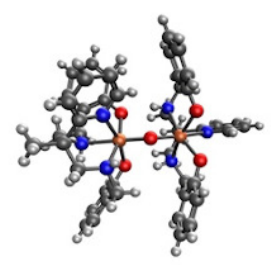

Complex 6: JOJGAS

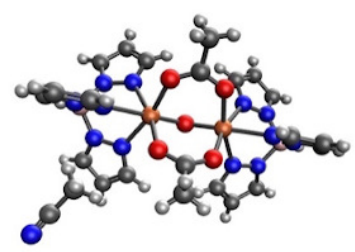

Complex 10: CACZIP

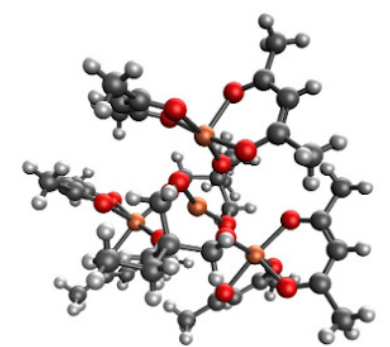

Complex 14: DUFNUO

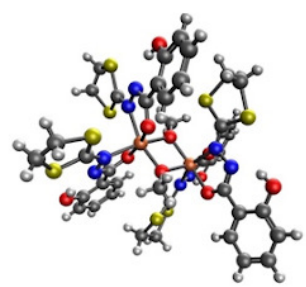

Complex 3: QOHVUF

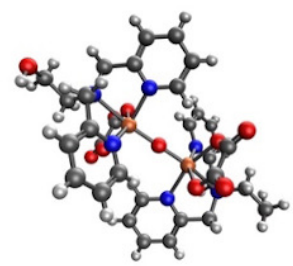

Complex 7: FAJQEO
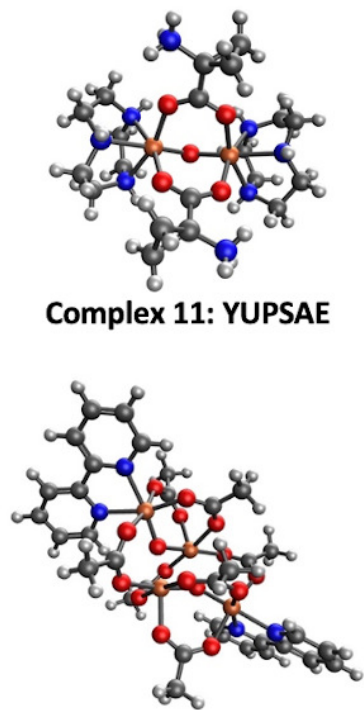

Complex 15: VIVDAF



Complex 4: ABIZOA



Complex 8: VIGGUN

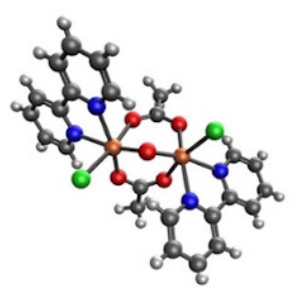

Complex 12: VABMUG

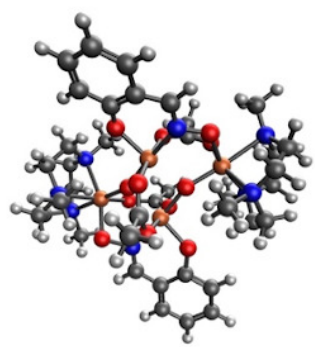

Complex 16: YAYPOD

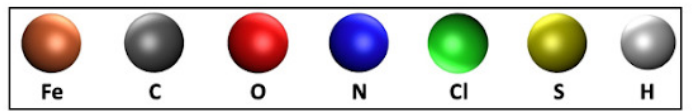

FIG. 3: Crystal structures of the binuclear (1-12) and tetranuclear (13-16) complexes with Fe (III) centers investigated in this study with their Cambridge structural database names.

binuclear and tetranuclear SMMs, respectively. We employed non-collinear formulation of SF-TDDFT[25]. To investigate the functional dependence, we used the following:

- Hybrid functionals: B3LYP[79], B5050LYP[24], PBE0[80], and PBE50[25].

- Minnesota functionals[81]: meta-GGA M06-L, and hybrid meta-GGA M06-2X.

- Range-separated hybrid functionals: CAM-B3LYP[82], LRC-wPBE[83], LRC$\omega \mathrm{PBEh}[84], \omega \mathrm{B} 97 \mathrm{X}-\mathrm{D}[85]$, and $\omega \mathrm{B} 97 \mathrm{M}-\mathrm{V}[86]$. 
We also evaluated basis-set effects (cc-pVDZ, def2-TZVP, and 6-31G(d,p)) and performance of effective core potentials (CRENBL, SBKJC, SRSC, and LANL2DZ).

\section{RESULTS AND DISCUSSION}

\section{A. Natural orbital analysis of mono- and bi-nuclear complexes}

TABLE I: Energy gaps ${ }^{a}(\Delta E, \mathrm{eV}),\left\langle S^{2}\right\rangle$ values $^{b}$, and density-based analysis of spin-flip states (denoted as "SF$n$ ") obtained from the high-spin hextet reference state (denoted as "ref.") in model mononuclear Fe (III) complexes ${ }^{c}$.

\begin{tabular}{lccccccccc}
\hline Complex & & ref. & $\mathrm{SF}_{1}$ & $\mathrm{SF}_{2}$ & $\mathrm{SF}_{3}$ & $\mathrm{SF}_{4}$ & $\mathrm{SF}_{5}$ & $\mathrm{SF}_{6}$ & $\mathrm{SF}_{7}$ \\
\multirow{2}{*}[\mathrm{Fe}(\mathrm{Cl})_{6}]{$^{3-}$} & $\left\langle S^{2}\right\rangle$ & 8.75 & 8.76 & 3.81 & 3.81 & 3.81 & 3.82 & 3.82 & 3.82 \\
& $\Delta E$ & & 0.00 & 1.61 & 1.61 & 1.61 & 1.73 & 1.74 & 1.74 \\
& $n_{u, n l}$ & & 5.00 & 3.16 & 3.19 & 3.04 & 3.03 & 3.09 & 3.08 \\
\hline \multirow{2}{*}{ ABI-m } & $\left\langle S^{2}\right\rangle$ & 8.77 & 8.80 & 3.85 & 3.84 & 3.85 & 3.83 & 3.83 & 3.84 \\
& $\Delta E$ & & 0.00 & 0.35 & 0.63 & 0.74 & 0.99 & 1.40 & 1.54 \\
& $n_{u, n l}$ & & 5.00 & 3.02 & 3.03 & 3.03 & 3.02 & 3.04 & 3.04 \\
\hline \multirow{2}{*}[\mathrm{Fe}(\mathrm{CN})_{6}]{$^{3-}{ }^{3-}$} & $\left\langle S^{2}\right\rangle$ & 8.76 & 3.83 & 3.82 & 3.82 & 3.82 & 3.82 & 3.82 & 8.76 \\
& $\Delta E$ & & 0.00 & 0.04 & 0.04 & 0.18 & 0.19 & 0.19 & 1.59 \\
& $n_{u, n l}$ & & 3.01 & 3.01 & 3.01 & 3.01 & 3.05 & 3.05 & 5.00 \\
\hline
\end{tabular}

${ }^{a}$ The energy gaps are computed with respect to the lowest spin-flip state $\left(\mathrm{SF}_{1}\right) .{ }^{b}$ The ideal $\left\langle S^{2}\right\rangle$ values of high-spin $\left(S=\frac{5}{2}\right)$ and low-spin $\left(S=\frac{3}{2}\right)$ states are 8.75 and 3.75 , respectively. ${ }^{c}$ LRC- $\omega$ PBEh/6-31G(d,p).

TABLE II: Occupancies of frontier natural orbitals $\left(n=n_{\alpha}+n_{\beta}\right)$ in the lowest hextet (ideal $\left\langle S^{2}\right\rangle$ is 8.75) and quartet (ideal $\left\langle S^{2}\right\rangle$ is 3.75 ) state in mononuclear Fe (III) complexes.

\begin{tabular}{lcccccc}
\hline Complex & $\left\langle S^{2}\right\rangle$ & $\mathrm{NO}_{1}$ & $\mathrm{NO}_{2}$ & $\mathrm{NO}_{3}$ & $\mathrm{NO}_{4}$ & $\mathrm{NO}_{5}$ \\
\hline \multirow{2}{*}{$\left.\mathrm{Fe}(\mathrm{Cl})_{6}\right]^{3-}$} & 8.76 & 1.00 & 1.00 & 1.00 & 1.00 & 1.00 \\
\multirow{2}{*}{$\mathrm{ABI}-\mathrm{m}$} & 3.81 & 0.20 & 0.86 & 1.00 & 1.14 & 1.82 \\
& 8.80 & 1.00 & 1.00 & 1.00 & 1.00 & 1.00 \\
\multirow{2}{*}[\mathrm{Fe}(\mathrm{CN})_{6}]{$^{3-}$} & 3.85 & 0.05 & 1.00 & 1.00 & 1.00 & 1.96 \\
\hline
\end{tabular}

To investigate the electronic structure pattern of the Fe (III) ion in a ligand field of various strength, we begin by considering three model single-center Fe (III) systems: $\left[\mathrm{Fe}(\mathrm{Cl})_{6}\right]^{3-}$, ABI-m, and $\left[\mathrm{Fe}(\mathrm{CN})_{6}\right]^{3-}$. $\quad\left[\mathrm{Fe}(\mathrm{Cl})_{6}\right]^{3-}$ and $\left[\mathrm{Fe}(\mathrm{CN})_{6}\right]^{3-}$ represent weak- and strong-field cases, respectively. Starting with a high-spin hextet reference state, we perform SF-TDDFT calculations (using LRC- $\omega \mathrm{PBEh} / 6-31 \mathrm{G}(\mathrm{d}, \mathrm{p})$ ) and analyze the manifold of the computed 
$S-1$ electronic states. The choice of the level of theory (functional/basis set) is justified by our benchmark calculations described below (Section IVB). Here, our aim is to verify the applicability of our SF protocol in describing the electronic structure of the Fe (III) complexes within the strong field regime, where non-Hund configurations appear at low energy spoiling the $\mathrm{HDvV}$ model, and to determine, on the basis of the natural orbital analysis, whether the ABIZOA complex (and then other complexes shown in Fig 3) fall in the weak field or the strong field category.

Table I shows energy gaps $(\Delta E)$, the number of effectively unpaired electrons $\left(n_{u, n l}\right)$, and the eigenvalue of the $S^{2}$ operator $\left(\left\langle S^{2}\right\rangle\right)$ for the spin-flip states of each model system (energy gaps are reported with respect to the lowest SF state). The occupancies of the natural frontier orbitals for the lowest hextet and quartet states are reported in Table II. The respective natural orbitals are shown in the SI. $\left[\mathrm{Fe}(\mathrm{Cl})_{6}\right]^{3-}$ and $\left[\mathrm{Fe}(\mathrm{CN})_{6}\right]^{3-}$ exhibit two threefold degeneracies of the quartet states due to $\mathrm{O}_{\mathrm{h}}$ symmetry. As expected from the crystal-field theory, the hextet state with five unpaired electrons is the lowest SF state in $\left[\mathrm{Fe}(\mathrm{Cl})_{6}\right]^{3-}$. In contrast, in $\left[\mathrm{Fe}(\mathrm{CN})_{6}\right]^{3-}$ a low-spin quartet state appears $1.6 \mathrm{eV}$ below the high-spin sextet state. The ABI-m model system clearly shows a weak-field pattern, with the hextet state being the lowest. Due to lower local symmetry, the orbital and state degeneracies are lifted.



$\left[\mathrm{Fe}(\mathrm{Cl})_{6}\right]^{3-}$



ABI-m

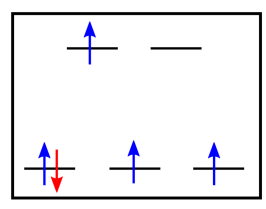

$\left[\mathrm{Fe}(\mathrm{CN})_{6}\right]^{3-}$

FIG. 4: Orbital occupations of the lowest state (i.e., $\mathrm{SF}_{1}$ ) in mononuclear Fe (III) complexes.

We use natural orbital occupancies (i.e, sum of $\alpha$ and $\beta$ NO occupations) of the lowest spin states to determine the nature of non-Hund configuration for mononuclear complexes and to establish the deviations from the HDvV model for multi-nuclear compounds. For the mononuclear complexes, the high-spin hextet states have all orbital occupancies that are nearly one, whereas in low-spin quartet states, the electrons become partially paired forming non-Hund configurations and the occupations of two of the NOs differ considerably from 1 (see Table II). Somewhat surprisingly, the difference between $\left[\mathrm{Fe}(\mathrm{Cl})_{6}\right]^{3-}$ and $\left[\mathrm{Fe}(\mathrm{CN})_{6}\right]^{3-}$ is not very large, although both $n_{u, n l}$ and the orbital occupations show the expected trend- 
larger contributions from the non-Hund configurations in the strong-field case. For multinuclear compounds, the appearance of non-Hund and even ionic configurations results in non-Heisenberg behavior, which requires mapping to biquadratic Heisenberg and Hubbard models to capture their magnetic behavior. Below we extend such natural orbital analysis to the binuclear complexes to assess whether the fit of magnetic susceptibility measurements to the HDvV model is appropriate for these systems.

TABLE III: Frontier natural orbital occupancies $\left(n=n_{\alpha}+n_{\beta}\right)$ and $n_{u, n l}$ of the lowest $S=4$ state showing an increase of ionic character with the absolute value of $J\left(\mathrm{~cm}^{-1}\right)$ in binuclear Fe (III) complexes.

\begin{tabular}{cccccccccccccc}
\hline Complex & $J$-exp & $J^{a}$-theo $^{b}$ & $n_{u, n l}$ & $\mathrm{NO}_{1}$ & $\mathrm{NO}_{2}$ & $\mathrm{NO}_{3}$ & $\mathrm{NO}_{4}$ & $\mathrm{NO}_{5}$ & $\mathrm{NO}_{6}$ & $\mathrm{NO}_{7}$ & $\mathrm{NO}_{8}$ & $\mathrm{NO}_{9}$ & $\mathrm{NO}_{10}$ \\
\hline 1 & -6.4 & -7.1 & 9.99 & 0.96 & 0.98 & 0.98 & 0.99 & 1.00 & 1.00 & 1.01 & 1.01 & 1.02 & 1.04 \\
2 & -13.6 & -16.6 & 9.98 & 0.94 & 0.97 & 0.98 & 0.98 & 0.99 & 1.01 & 1.02 & 1.02 & 1.03 & 1.06 \\
3 & -13.7 & -15.7 & 9.98 & 0.94 & 0.97 & 0.97 & 0.98 & 0.99 & 1.01 & 1.02 & 1.03 & 1.03 & 1.06 \\
4 & -21.3 & -20.4 & 9.98 & 0.94 & 0.96 & 0.98 & 0.98 & 0.99 & 1.01 & 1.02 & 1.02 & 1.03 & 1.06 \\
5 & $-100^{c}$ & -106.8 & 9.91 & 0.90 & 0.91 & 0.93 & 1.00 & 1.00 & 1.00 & 1.00 & 1.07 & 1.09 & 1.10 \\
6 & -87.5 & -91.9 & 9.92 & 0.89 & 0.93 & 0.93 & 1.00 & 1.00 & 1.00 & 1.00 & 1.07 & 1.07 & 1.10 \\
7 & -98 & -100.7 & 9.91 & 0.89 & 0.93 & 0.93 & 1.00 & 1.00 & 1.00 & 1.00 & 1.07 & 1.07 & 1.11 \\
8 & -108 & -105.4 & 9.90 & 0.89 & 0.92 & 0.93 & 1.00 & 1.00 & 1.00 & 1.00 & 1.07 & 1.07 & 1.11 \\
9 & -119 & -109.8 & 9.90 & 0.89 & 0.90 & 0.93 & 0.99 & 1.00 & 1.01 & 1.01 & 1.07 & 1.09 & 1.11 \\
10 & -121 & -124.7 & 9.88 & 0.88 & 0.90 & 0.92 & 0.99 & 0.99 & 1.01 & 1.01 & 1.08 & 1.10 & 1.12 \\
11 & -130 & -123.0 & 9.88 & 0.88 & 0.91 & 0.92 & 0.99 & 0.99 & 1.01 & 1.01 & 1.08 & 1.09 & 1.12 \\
12 & -132 & -119.4 & 9.88 & 0.88 & 0.91 & 0.92 & 0.99 & 0.99 & 1.01 & 1.01 & 1.08 & 1.09 & 1.12 \\
\hline${ }^{a}$ Experimental exchange coupling from Refs. [61]- $[76]$. & ${ }^{b}$ Computed exchange coupling using \\
LRC- $\omega \mathrm{PBEh} / 6-31 \mathrm{G}(\mathrm{d}, \mathrm{p})$ & and the Landé interval rule. ${ }^{c}$ The exchange coupling is taken to
\end{tabular}

Table III show the results for the set of binuclear Fe (III) SMMs (see Fig. 3 for structures). In all of them, the high-spin $S=5$ states have near one orbital occupancy for all 10 frontier natural orbitals, with expected $n_{u, n l}=10$. In contrast, the lower $S=4$ states show slight deviations of occupations from one, which increase in the higher exchange coupling regime, resulting in larger discrepancies between the theoretical (of this work) and experimental (see Refs. [61]-[76]) exchange couplings. The same trend is reported for the number of unpaired electrons, which decreases from 9.99 to 9.88 upon an increase in exchange interaction. The effects of a large magnitude of exchange coupling can be compared to that of the lowest SF state in $\left[\mathrm{Fe}(\mathrm{CN})_{6}\right]^{3-}$, where a large crystal field splitting stabilizes the non-Hund states (Fig. $4)$.

Overall, we observed small discrepancy between experimentally derived $J$-values and the ones extracted from $a b$ initio calculation using Eq. (3). This deviation increases with the 
size of $J$. Additionally, the larger is the $J$ coupling (in absolute value) and the larger is the deviation of $n_{u, n l}$ from 10 and of the NO occupancies from 1, suggesting the presence of ionic and non-Hund contributions. These configurations are responsible for the discrepancy between theory and experiment in the higher anti-ferromagnetic regime (i.e., more negative $J$-values). However, deviation of $n_{u, n l}$ from 10 (less than 2\%) and of NO occupancies from unity (within $8 \%$ ) is small, meaning that the HDvV model can be considered valid for the binuclear Fe (III) compounds under study.

\section{B. Benchmark calculations}

Having established a procedure to validate the HDvV model, we now proceed to benchmarking the functional and basis-set choice against experiments. For the binuclear Fe (III) SMMs, SF-TDDFT calculations using high-spin reference of $S=5$ yield $S=5$ and $S=4$ states of $M_{s}=4$ components from which the exchange couplings are computed using Eq. (3). The $\left\langle S^{2}\right\rangle$ values for the spin-flipped $S=5$ and $S=4$ states are close to 30 and 20 values respectively, with negligible spin contamination ranging between 0.01 and 0.06 . We compute statistical measures of errors such as mean absolute error (MAE), mean error (ME), and standard deviation error (SDE) of the computed $J$-values with respect to the experimentally derived ones.

TABLE IV: $J$-couplings $\left(\mathrm{cm}^{-1}\right)$ for 12 binuclear Fe(III) systems computed using NC-SF-TDDFT with selected functionals and the $6-31 \mathrm{G}(\mathrm{d}, \mathrm{p})$ basis set.

\begin{tabular}{|c|c|c|c|c|c|c|c|c|c|c|c|c|}
\hline plex & Exp. & $3 \mathrm{~L}$ & LYP & & & & & $\begin{array}{l}\text { CAM- } \\
\text { B3LYP }\end{array}$ & $\begin{array}{l}\text { LRC- } \\
\omega \mathrm{PBE}\end{array}$ & $\begin{array}{l}\text { LRC- } \\
\omega \text { PBEh }\end{array}$ & $\begin{array}{c}\omega \mathrm{B} 97 \\
\text { X-D }\end{array}$ & $\begin{array}{l}\omega \mathrm{B} 97 \\
\mathrm{M}-\mathrm{V}\end{array}$ \\
\hline 1 & -6.4 & -10.9 & -3.6 & -6.9 & -2.7 & -11.5 & -5.3 & -7.7 & -8.2 & -7.1 & -7.3 & -7.7 \\
\hline 2 & -13.6 & -24.7 & -8.5 & -16.9 & -7.2 & -25.5 & -11.9 & -19 & -22.8 & -16.6 & -18.1 & -18.4 \\
\hline 3 & -13.7 & -23.5 & -6.3 & -15.2 & -5 & -28.5 & -10.8 & -17.6 & -23.6 & -15.7 & -13.4 & -17.4 \\
\hline 4 & -21.3 & -27.3 & -11.7 & -20 & -10 & -31.9 & -6.5 & -21.1 & -27.8 & -20.4 & -22.3 & -23 \\
\hline 5 & -100.0 & -134.2 & -70.2 & -101.9 & -61.8 & -129.9 & -163.4 & -119.5 & -139.5 & -106.8 & -116.1 & -115.5 \\
\hline 6 & -87.5 & -115.7 & -59.4 & -87.4 & -52.7 & -125.3 & -44.2 & -103.2 & -122.4 & -91.9 & -99.9 & -101.5 \\
\hline 7 & -98.0 & -124.1 & -66.6 & -95.6 & -58.8 & -119.6 & -90.8 & -113.1 & -133.2 & -100.7 & -109.3 & -111 \\
\hline 8 & -108.4 & -140.3 & -64.7 & -102.4 & -57.3 & -147.4 & -104 & -118 & -142 & -105.4 & -114.6 & -112.1 \\
\hline 9 & -119.0 & -147.3 & -67 & -106.9 & -59 & -155.2 & -96.5 & -123.5 & -148.8 & -109.8 & -119.3 & -118 \\
\hline 10 & -121.0 & -164.7 & -74.8 & -119.9 & -66.1 & -172.8 & -56.9 & -140.4 & -171.2 & -124.7 & -136.5 & -132.1 \\
\hline 11 & -130.5 & -166.3 & -77.1 & -120.1 & -66.9 & -170.3 & -173.2 & -139.2 & -166.1 & -123.1 & -134. & -131.9 \\
\hline 12 & -132.0 & -155.9 & -74 & -114.5 & -65 & -163.7 & -87.4 & -134.7 & -162 & -119.4 & -130.5 & -130.6 \\
\hline MAE & & 23.7 & 30.6 & 4.9 & 36.6 & 27.5 & 26.0 & 8.8 & 26.4 & 4.7 & 6.1 & 6.0 \\
\hline $\mathrm{ME}$ & & -23.7 & 30.6 & 3.6 & 36.6 & -27.5 & 8.4 & -8.8 & -26.4 & 0.8 & -5.8 & -5.6 \\
\hline SDE & & 12.8 & 20.4 & 6.5 & 23.7 & 28.13 & 35.4 & 7.0 & 15.4 & 6.0 & 11.48 & 6.0 \\
\hline
\end{tabular}




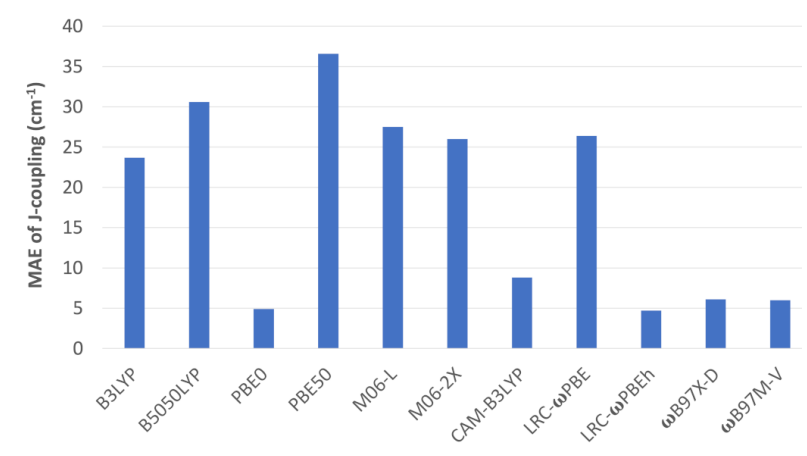

FIG. 5: Mean absolute error (MAE) of $J$-couplings calculated using different functionals with respect to experimental values for 12 binuclear Fe (III) systems. The 6-31G(d,p) basis set was used for all atoms.

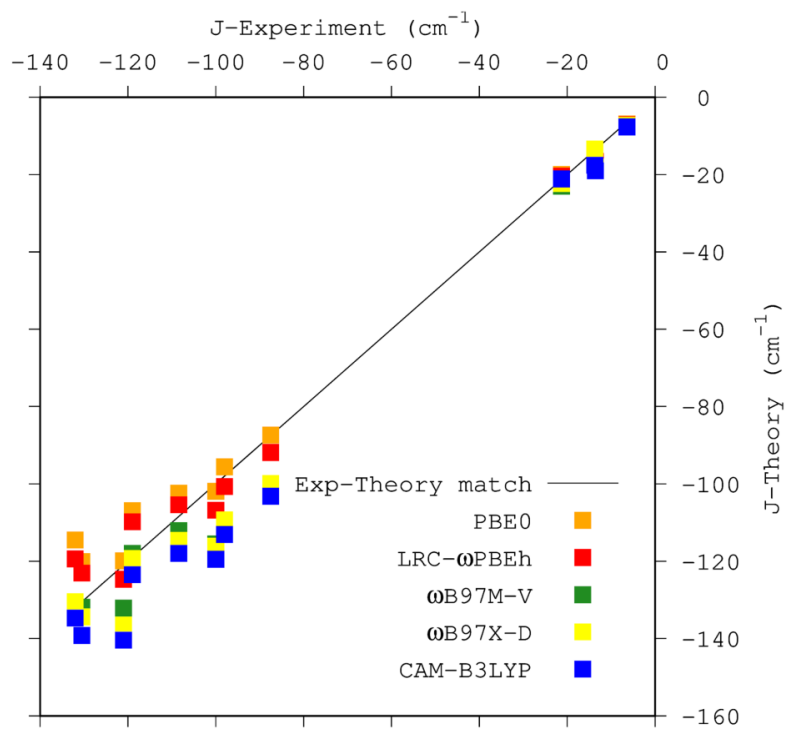

FIG. 6: Theoretical versus experimental $J$-couplings for functionals with MAE $<10 \mathrm{~cm}^{-1}$. The blue line shows the perfect match.

Tables IV collect the results for selected functionals using the 6-31G(d,p) basis set on all atoms; the respective MAE are shown graphically in Fig. 5. Range-separated hybrid functionals with short-range Hartree-Fock exchange such as LRC- $\omega$ PBEh, $\omega$ B97M-V, $\omega$ B97X-D, and CAM-B3LYP provide a good estimate of exchange interactions (with MAEs around 5 $\mathrm{cm}^{-1}$ ). PBE0 also performs well. In contrast, M06-L, LRC- $\omega$ PBE, B3LYP, CAM-B3LYP, $\omega \mathrm{B} 97 \mathrm{X}-\mathrm{D}$, and $\omega \mathrm{B} 97 \mathrm{M}-\mathrm{V}$ functionals underestimate the couplings whereas the other functionals overestimate them. Hybrid functionals PBE50, B5050LYP, B3LYP, and Minnesota06 functionals have MAEs greater than $20 \mathrm{~cm}^{-1}$, to be compared with MAEs below $10 \mathrm{~cm}^{-1}$ for range-separated functionals. Fig. 6 provides a more detailed view of this trend by show- 
ing the theoretical and experimental couplings as a scatter plot for the five best-performing functionals (LRC- $\omega$ PBEh, $\omega$ B97M-V, $\omega$ B97X-D, CAM-B3LYP, and PBE0). All of them reproduce the experimental observation: the connectivity between the iron atoms plays a crucial rule in tuning the exchange interaction. Dibridged oxo units feature low couplings, whereas a single oxo bridge or oxo bridge paired with acetate bridges result in higher $J$ values.

TABLE V: Basis-set dependence of $J$-couplings $\left(\mathrm{cm}^{-1}\right)$ for the 12 binuclear complexes computed with NC-SF-TDDFT with $\omega$ PBEh.

\begin{tabular}{ccccccccc}
\hline Complex & Exp. & cc-pVDZ & $\begin{array}{c}\text { def2- } \\
\text { TZVP }\end{array}$ & $\begin{array}{c}6-31 \mathrm{G} \\
(\mathrm{d}, \mathrm{p})\end{array}$ & $\begin{array}{c}\text { CREN } \\
\text { BL }\end{array}$ & $\begin{array}{c}\text { SBK } \\
\text { JC }\end{array}$ & SRSC & $\begin{array}{c}\text { LANL } \\
2 \mathrm{DZ}\end{array}$ \\
\hline 1 & -6.4 & -5.9 & -5.8 & -7.1 & -5.3 & -5.5 & -6.0 & -7.2 \\
2 & -13.6 & -16.1 & -15.9 & -16.6 & -14.5 & -15.0 & -15.2 & -19.0 \\
3 & -13.7 & -15.1 & -14.5 & -15.7 & -13.9 & -14.2 & -14.4 & -17.5 \\
4 & -21.3 & -20.2 & -19.4 & -20.4 & -18.9 & -18.8 & -18.9 & -21.9 \\
5 & -100.0 & -108.8 & -108.2 & -106.8 & -101.0 & -99.4 & -101.8 & -115.2 \\
6 & -87.5 & -91.4 & -92.3 & -91.9 & -83.6 & -84.2 & -84.1 & -96.5 \\
7 & -98.0 & -103.3 & -104.6 & -100.7 & -94.8 & -93.6 & -96.8 & -109.8 \\
8 & -108.4 & -104.7 & -105.4 & -105.4 & -93.9 & -92.2 & -126.3 & -146.6 \\
9 & -119.0 & -109.4 & -109.9 & -109.8 & -96.3 & -95.5 & -97.7 & -111.7 \\
10 & -121.0 & -122.0 & -123.2 & -124.7 & -109.1 & -107.2 & -109.9 & -127.2 \\
11 & -130.5 & -122.3 & -123.2 & -123.1 & -109.2 & -108.8 & -109.8 & -127.1 \\
12 & -132.0 & -118.3 & -119.4 & -119.4 & -106.4 & -104.8 & -140.3 & -160.9 \\
\hline MAE & & 5.0 & 4.9 & 4.7 & 9.1 & 9.7 & 7.6 & 10.9 \\
ME & & 1.2 & 0.8 & 0.8 & 8.7 & 9.4 & 2.5 & -9.1 \\
SDE & & 6.6 & 6.4 & 6.0 & 10.0 & 10.5 & 11.1 & 13.1 \\
\hline
\end{tabular}

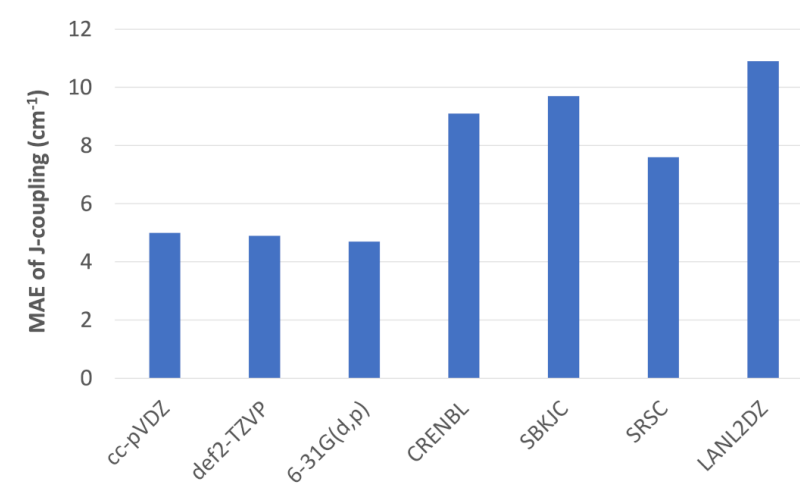

FIG. 7: MAE of the $J$-couplings computed using different basis sets with LRC- $\omega$ PBEh functional for the 12 iron (III) binuclear systems.

To find optimal computational setting to be used for binuclear and tetranuclear Fe (III) systems, we proceed to investigate the effects of the basis set and effective core potentials 
(ECPs) when coupled with one of the best performing functional, i.e., the LRC- $\omega$ PBEh functional. Fig. 7 shows the results obtained using the 6-31G(d,p), cc-pVDZ, and def2TZVP basis sets (see also Table V). The calculated $J$ values are not sensitive to the basisset choice, and agree well with experimental values with MAE of less than $5 \mathrm{~cm}^{-1}$. In contrast, ECPs perform poorly with MAE of around $10 \mathrm{~cm}^{-1}$ and high standard deviations. We conclude that LRC- $\omega \mathrm{PBEh} / 6-31 \mathrm{G}(\mathrm{d}, \mathrm{p})$ is the best computational setting for computing exchange interactions in these Fe(III) SMMs, which is consistent with findings by Orms and Krylov for binuclear copper SMMs[28].

\section{Tetranuclear Fe (III) complexes}
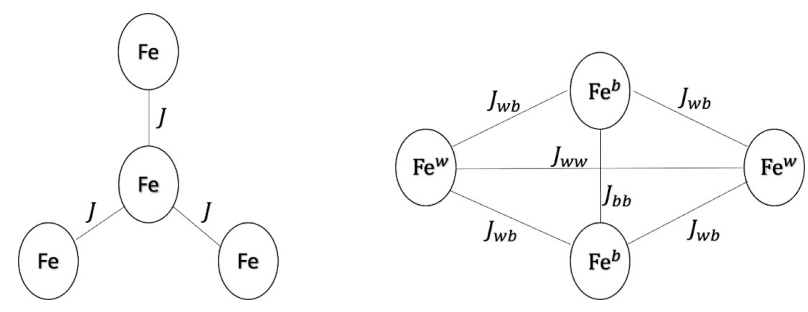

FIG. 8: Iron core in star-type complexes 13, 14 (left) and butterfly-type complexes 15, 16 (right) showing different exchange interactions. The superscripts $w$ and $b$ denote wing and body iron atoms, respectively.

Having validated the SF-based computational protocol, we proceed to investigate the more-challenging tetranuclear Fe (III) SMMs. We note that accurate determination of exchange constants is important in multinuclear complexes, because small differences can change the ground state spin of the molecule[87].

To tackle poly-nuclear complexes, we follow the framework developed by Mayhall and Head-Gordon[36]. First, we perform a single SF calculation providing energies and eigenstates, which are projected onto the neutral determinant basis, following Ref. [36]. SFTDDFT calculations using high-spin reference of $S=10$ yield $S=9$ states whose $\left\langle S^{2}\right\rangle$ values are close to 90 showing small spin contamination (between 0.02 and 0.38 ). Second, we extract exchange constants in tetranuclear single-molecule magnets (complexes 13-16 in Fig. 3) as prescribed by Mayhall's approach by mapping the effective Hamiltonian constructed from single spin-flipped eigenstates and their eigenvalues to the $\mathrm{HDvV}$ model. Additional details on Mayhall's approach (translated into a post-processing Python script) and a sam- 
ple input for running Q-Chem calculations when combined with the parameterization of the HDvV Hamiltonian are given in the SI. Complexes 13 and 14 have star-like structures and the experimental fits show a predominant exchange interaction between the central and three peripheral irons. Complexes 15 and 16 feature a butterfly-like core, with dominant wing-body interactions $\left(J_{w b}\right)$ and much smaller body-body $\left(J_{b b}\right)$ and wing-wing $\left(J_{w w}\right)$ interactions. In fitting the experimental data, $J_{w w}$ is often neglected. The effective Hamiltonian approach enables the determination of all individual exchange interactions without assuming simplified $\mathrm{HDvV}$ models, as often done in the experimental fitting.

TABLE VI: $J$-couplings $\left(\mathrm{cm}^{-1}\right)$ for tetranuclear iron (III) complexes computed with NC-SFTDDFT/LRC- $\omega$ PBEh/6-31G(d,p).

\begin{tabular}{cccc}
\hline Complex & Exp. & LRC- $\omega$ PBEh $/ 6-31 \mathrm{G}(\mathrm{d}, \mathrm{p})$ & ave $J^{a}$ \\
\hline 13 & -8.3 & $-10.1 ;-11.4 ;-11.2$ & -10.9 \\
14 & -8.5 & $-10.6 ;-11.7 ;-11.7$ & -11.3 \\
15 & $-45.5 ;-8.9$ & $-43.7 ;-42.3 ;-43.3 ;-44.5 ;-3.5 ; 5.1^{b}$ & $-43.5 ; 0.8$ \\
16 & $-46 ; 0.0$ & $-27.8 ;-30.1 ;-45.7 ;-58.6 ;-1.0 ;-1.9^{b}$ & $-40.6 ;-1.5$ \\
\hline
\end{tabular}

a "Ave" stands for the average of $J$ values extracted using LRC- $\omega$ PBEh/6-31G(d,p). ${ }^{b}$ The bigger $J$-values refer to the four wing-body couplings, while the smaller $J$-values are the body-body and wing-wing ones.

Table VI shows the results. In complex 16, a simple two- $J$ model (assumed in the experimental study) appears to be insufficient. The experimental fitting assumed all wing-body interactions to be the same, but our calculations show that the wing-body interactions can differ by up to $30 \mathrm{~cm}^{-1}$. Still, the average $J$ 's matches the experimental coupling. In other complexes, the simplified $\mathrm{HDvV}$ models appear to be justified. Overall, our calculations closely reproduce the dominant interactions between the iron centers with an error of less than $6 \mathrm{~cm}^{-1}$. For the lowest target $S=9$ states, the occupations of the frontier NOs $\left(n_{\alpha}+n_{\beta}\right)$ is close to 1 and the deviation of the number of unpaired electrons $\left(n_{u, n l}\right)$ from 20 is small, i.e., less than 0.04, accordingly with the low exchange interaction regime for which the HDvV model is a good approximation. Therefore, such calculations and the NOs analysis of this work can help validating the simplified HDvV models used for fitting macroscopic properties of poly-nuclear transition-metal complexes. 


\section{CONCLUSIONS}

In this contribution, we investigated magnetic properties of 12 binuclear and four tetranuclear Fe (III) SMMs. We employed NC-SF-TDDFT method to parameterize HDvV Hamiltonian. The validity of this approach was assessed using natural orbital analysis, which shows that the target spin-flip states in all complexes are within the HDvV domain, corresponding to the weak field regime. Spin-contamination is negligible in all these complexes. We employed several representative functionals (hybrid, Minnesota, range-separated hybrids), basis sets, and ECPs. In agreement with earlier studies for Cu (II) SMMs[28], range-separated hybrid functionals with short-range Hartree-Fock exchange agree well with experiments (MAE $\left.<10 \mathrm{~cm}^{-1}\right)$. We recommend LRC- $\omega \mathrm{PBEh} / 6-31 \mathrm{G}(\mathrm{d}, \mathrm{p})$ for computing $J_{-}$ couplings using NC-SF-TDDFT. In poly-nuclear SMMs, the couplings extracted from the $a b$ initio-parameterized effective Hamiltonians reliably describe interactions between all metal centers, without any simplifying assumptions. Such calculations can be used for a priori determination of the validity of simplified HDvV models. The effective Hamiltonian approach combined with SF-TDDFT can be extended to study even larger SMMs. [88, 89] Our results underscore the role of the connectivity between the metal centers in tuning the exchange interactions in SMMs.

\section{Acknowledgments}

We thank Dr. Pavel Pokhilko and Dr. Sven Kähler for their help and guidance in the initial stages of this work.

This work is supported by the Department of Energy through the DE-SC0018910 grant.

The authors declare the following competing financial interest(s): A.I.K. is the president and a part-owner of Q-Chem, Inc.

\section{Supplementary Information}

Multiple-center molecular magnets: Mayhall's approach, structures of mononuclear systems, natural orbitals in mononuclear systems, sample input for effective Hamiltonian cal- 
culations. 
[1] D. Gatteschi, R. Sessoli, and J. Villain, Molecular Nanomagnets. Oxford University Press: Oxford, U.K., 2006.

[2] M.R. Pederson and T. Baruah, Molecular magnets: Phenomenology and theory, in Handbook of Magnetism and Magnetic Materials, edited by S. Parkin, pages 1-22. J. Wiley and Sons, London, 2007.

[3] M. N. Leuenberger and D. Loss, Quantum computing in molecular magnets, Nature 410, 789 (2001).

[4] L. Bogani and W. Wernsdorfer, Molecular spintronics using single-molecule magnets, Nat. Mater. 7, 179 (2008).

[5] M. D. Jenkins, D. Zueco, O. Roubeau, G. Aromi, J. Majere, and F. Luis, A scalable architecture for quantum computation with molecular nanomagnets, Dalton Trans. 45, 16682 (2016).

[6] F. Neese and D. A. Pantazis, What is not required to make a single molecule magnet, Faraday Discuss. 148, 229 (2011).

[7] S. Demir, J. M. Zadrozny, M. Nippe, and J. R. Long, Exchange coupling and magnetic blocking in bipyrimidyl radical-bridged dilanthanide complexes, J. Am. Chem. Soc. 134, 18546 (2012).

[8] T. Gupta and G. Rajaraman, Modelling spin hamiltonian parameters of molecular nanomagnets, Chem. Comm. 52, 8972 (2016).

[9] C. Schlegel, J. van Slageren, M. Manoli, E. K. Brechin, and M. Dressel, Direct observation of quantum coherence in single-molecule magnets, Phys. Rev. Lett. 101, 147203 (2008).

[10] A. L. Barra, A. Caneschi, A. Cornia, F. Fabrizi de Biani, D. Gatteschi, C. Sangregorio, R. Sessoli, and L. Sorace, Single-molecule magnet behavior of a tetranuclear iron (III) complex. The origin of slow magnetic relaxation in iron (III) clusters, J. Am. Chem. Soc. 121, 5302 (1999).

[11] A. K. Boudalis, Y. Sanakis, J. M. Clemente-Juan, B. Donnadieu, V. Nastopoulos, A. Mari, Y. Coppel, J.-P. Tuchagues, and S. P. Perlepes, A family of enneanuclear iron(II) singlemolecule magnets, Chem. Eur. J. 14, 2514 (2008).

[12] G. Christou, Single-molecule magnets: a molecular approach to nanoscale magnetic materials, Polyhedron 24, 2065 (2005). 
[13] M. Mannini, F. Pineider, P. Sainctavit, C. Danieli, E. Otero, C. Sciancalepore, A. M. Talarico, M. Arrio, A. Cornia, D. Gatteschi, and R. Sessoli, Magnetic memory of a single-molecule quantum magnet wired to a gold surface, Nature 8, 194 (2009).

[14] J. Finley, P.-Å. Malmqvist, B. O. Roos, and L. Serrano-Andrés, The multi-state CASPT2 method, Chem. Phys. Lett. 288, 299 (1998).

[15] C. Angeli, R. Cimiraglia, S. Evangelisti, T. Leininger, and J.-P. Malrieu, Introduction of n-electron valence states for multireference perturbation theory, J. Chem. Phys. 114, 10252 (2001).

[16] M. Atanasov, D. Aravena, E. Suturina, E. Bill, D. Maganas, and F. Neese, First prinicples approach to the electronic structure, magnetic anisotropy and spin relaxation in mononuclear 3d-transition metal single molecular magnets, Coord. Chem. Rev. 289-290, 177 (2015).

[17] L. F. Chibotaru, Theoretical Understanding of Anisotropy in Molecular Nanomagnets, pages 185-229. Springer Berlin Heidelberg, Berlin, Heidelberg, 2015.

[18] L. Noodleman, Valence bond description of antiferromagnetic coupling in transition metal dimers, J. Chem. Phys. 74, 5737 (1981).

[19] S. Sinnecker, F. Neese, L. Noodleman, and W. Lubitz, Calculating the electron paramagnetic resonance parameters of exchange coupled transition metal complexes using broken symmetry density functional theory: application to a $\mathrm{Mn}(\mathrm{III}) / \mathrm{Mn}(\mathrm{IV})$ model compound, J. Am. Chem. Soc. 126, 2613 (2004).

[20] I. P. R. Moreira; R. Costa; M. Filatov; F. Illas, Restricted ensemble-referenced Kohn-Sham versus broken symmetry approaches in density functional theory: Magnetic coupling in $\mathrm{Cu}$ binuclear complexes, J. Chem. Theory Comput. 3, 764 (2007).

[21] A. I. Krylov, The spin-flip equation-of-motion coupled-cluster electronic structure method for a description of excited states, bond-breaking, diradicals, and triradicals, Acc. Chem. Res. 39, 83 (2006).

[22] A. I. Krylov, Size-consistent wave functions for bond-breaking: The equation-of-motion spinflip model, Chem. Phys. Lett. 338, 375 (2001).

[23] S. V. Levchenko and A. I. Krylov, Equation-of-motion spin-flip coupled-cluster model with single and double substitutions: Theory and application to cyclobutadiene, J. Chem. Phys. 120, 175 (2004).

[24] Y. Shao, M. Head-Gordon, and A. I. Krylov, The spin-flip approach within time-dependent 
density functional theory: Theory and applications to diradicals, J. Chem. Phys. 118, 4807 (2003).

[25] Y. A. Bernard, Y. Shao, and A. I. Krylov, General formulation of spin-flip time-dependent density functional theory using non-collinear kernels: Theory, implementation, and benchmarks, J. Chem. Phys. 136, 204103 (2012).

[26] D. Lefrancois, M. Wormit, and A. Dreuw, Adapting algebraic diagrammatic construction schemes for the polarization propagator to problems with multi-reference electronic ground states exploiting the spin-flip ansatz, J. Chem. Phys. 143, 124107 (2015).

[27] M. de Wergifosse, C. Bannwarth, and S. Grimme, A simplified spin-flip time-dependent density functional theory approach for the electronic excitation spectra of very large diradicals, J. Phys. Chem. A 123, 5815 (2019).

[28] N. Orms and A. I. Krylov, Singlet-triplet energy gaps and the degree of diradical character in binuclear copper molecular magnets characterized by spin-flip density functional theory, Phys. Chem. Chem. Phys. 20, 13127 (2018).

[29] M. Alessio and A. I. Krylov, Equation-of-motion coupled-cluster protocol for calculating magnetic properties: Theory and applications to single-molecule magnets, J. Chem. Theory Comput. 17, 4225 (2021).

[30] F. Wang and T. Ziegler, Time-dependent density functional theory based on a noncollinear formulation of the exchange-correlation potential, J. Chem. Phys. 121, 12191 (2004).

[31] J. P. Malrieu, R. Caballol, C. J. Calzado, C. de Graaf, and N. Guihéry, Magnetic interactions in molecules and highly correlated materials: Physical content, analytical derivation, and rigorous extraction of magnetic Hamiltonians, Chem. Rev. 114, 429 (2013).

[32] N. Orms, D. R. Rehn, A. Dreuw, and A. I. Krylov, Characterizing bonding patterns in diradicals and triradicals by density-based wave function analysis: A uniform approach, J. Chem. Theory Comput. 14, 638 (2018).

[33] R. Valero, F. Illas, and D. G. Truhlar, Magnetic coupling in transition-metal binuclear complexes by spin-flip time-dependent density functional theory, J. Chem. Theory Comput. 7, 3523 (2011).

[34] H. R. Zhekova, M. Seth, and T. Ziegler, Calculation of the exchange coupling constants of copper binuclear systems based on spin-flip constricted variational density functional theory, J. Chem. Phys. 135, 184105 (2012). 
[35] R. P. Joshi, J. J. Phillips, K. J. Mitchell, G. Christou, K. A. Jackson, and J. E. Peralta, Accuracy of density functional theory methods for the calculation of magnetic exchange couplings in binuclear iron(III) complexes, Polyhedron 176, 114194 (2020).

[36] N. J. Mayhall and M. Head-Gordon, Computational quantum chemistry for multiple-site Heisenberg spin couplings made simple: Still only one spin-flip required, J. Phys. Chem. Lett. 6, $1982(2015)$.

[37] D. Casanova and A. I. Krylov, Spin-flip methods in quantum chemistry, Phys. Chem. Chem. Phys. 22, 4326 (2020).

[38] L. V. Slipchenko and A. I. Krylov, Singlet-triplet gaps in diradicals by the spin-flip approach: A benchmark study, J. Chem. Phys. 117, 4694 (2002).

[39] L. V. Slipchenko and A. I. Krylov, Electronic structure of the 1,3,5,-tridehydrobenzene triradical in its ground and excited states, J. Chem. Phys. 118, 9614 (2003).

[40] L. V. Slipchenko, T. E. Munsch, P. G. Wenthold, and A. I. Krylov, 5-dehydro-1,3quinodimethane: A hydrocarbon with an open-shell doublet ground state, Angew. Chem., Int. Ed. 43, 742 (2004).

[41] A. M. C. Cristian, Y. Shao, and A. I. Krylov, Bonding patterns in benzene triradicals from structural, spectroscopic, and thermochemical perspectives, J. Phys. Chem. A 108, 6581 (2004).

[42] A. I. Krylov, Triradicals, J. Phys. Chem. A 109, 10638 (2005).

[43] B. G. Levine, C. Ko, J. Quenneville, and T. J. Martínez, Conical intersections and double excitations in time-dependent density functional theory, Mol. Phys. 104, 1039 (2006).

[44] D. Lefrancois, D. Tuna, T. J. Martínez, and A. Dreuw, The spin-flip variant of the algebraicdiagrammatic construction yields the correct topology of $\mathrm{S}_{1} / \mathrm{S}_{0}$ conical intersections, J. Chem. Theory Comput. 13, 4436 (2017).

[45] N. Minezawa and M. S. Gordon, Optimizing conical intersections by spin-flip density functional theory: Application to ethylene, J. Phys. Chem. A 113, 12749 (2009).

[46] X. Zhang and J. M. Herbert, Analytic derivative couplings for spin-flip configuration interaction singles and spin-flip time-dependent density functional theory, J. Chem. Phys. 141, 064104 (2014).

[47] S. Gozem, A. I. Krylov, and M. Olivucci, Conical intersection and potential energy surface features of a model retinal chromophore: Comparison of eom-cc and multireference methods, 
J. Chem. Theory Comput. 9, 284 (2013).

[48] C. de Graaf and R. Broer, Magnetic interactions in molecules and solids. Springer, 2016.

[49] W. Heisenberg, Zur Theorie des Ferromagnetismus, Z. Phys. 49, 619 (1928).

[50] P. A. M. Dirac, On the theory of quantum mechanics, Proc. R. Soc. London A 112, 661 (1926).

[51] J. H. van Vleck, The Theory of Electric and Magnetic Susceptibilities. Clarendon Press, Oxford, 1932.

[52] B. Bleaney and K.D. Bowers, Anomalous paramagnetism of copper acetate, Proc. R. Soc. London, Ser. A 214, 451 (1952).

[53] N. J. Mayhall and M. Head-Gordon, Computational quantum chemistry for single Heisenberg spin couplings made simple: Just one spin flip required, J. Chem. Phys. 141, 134111 (2014).

[54] A. Landé, Termstruktur und Zeemaneffekt der Multipletts, Z. Phys. 15, 189 (1923).

[55] P. Pokhilko and A. I. Krylov, Effective Hamiltonians derived from equation-of-motion coupledcluster wave-functions: Theory and application to the Hubbard and Heisenberg Hamiltonians, J. Chem. Phys. 152, 094108 (2020).

[56] P.-O. Löwdin, Quantum theory of many-particle systems. I. Physical interpretations by means of density matrices, natural spin-orbitals, and convergence problems in the method of configurational interaction, Phys. Rev. 97, 1474 (1955).

[57] F. Plasser, M. Wormit, and A. Dreuw, New tools for the systematic analysis and visualization of electronic excitations. I. Formalism, J. Chem. Phys. 141, 024106 (2014).

[58] A. I. Krylov, From orbitals to observables and back, J. Chem. Phys. 153, 080901 (2020).

[59] A. V. Luzanov, Effectively unpaired electrons for singlet states: from diatomics to graphene nanoclusters, in Practical Aspects of Computational Chemistry IV, edited by J. Leszczynski and M. Shukla, chapter 6, pages 151-206. Springer-Verlag, New York, 2016.

[60] M. Head-Gordon, Characterizing unpaired electrons from the one-particle density matrix, Chem. Phys. Lett. 372, 508 (2003).

[61] Y. Yahsi, H. Kara, L. Sorace, and O. Buyukgungor, Mono- and dinuclear Fe(III) complexes with the $\mathrm{N}_{2} \mathrm{O}_{2}$ donor 5-chlorosalicylideneimine ligands; synthesis, x-ray structural characterization and magnetic properties, Inorg. Chim. Acta 366, 191 (2011).

[62] F. Banse, V. Balland, C. Philouze, E. Riviere, L. Tchertanova, and J.-J. Girerd, Mono- and dinuclear Fe(III) complexes with the tridentate N-ethyl-N-(2-aminoethyl)salicylaldiminato lig- 
and. X-ray structures, magnetic and spectroscopic properties, Inorg. Chim. Acta 353, 223 (2003).

[63] N. Bouslimani, N. Clément, G. Rogez, P. Turek, M. Bernard, S. Dagorne, D. Martel, H. N. Cong, and R. Welter, Synthesis and magnetic properties of new mono- and binuclear iron complexes with salicyloylhydrazono dithiolane ligand, Inorg. Chem. 47, 7623 (2008).

[64] E.-Q. Gao, L.-H. Yin, J.-K. Tang, P. Cheng, D.-Z. Liao, Z.-H. Jiang, and S.-P. Yan, Synthesis, crystal structure and magnetic properties of a new dialkoxo-bridged diiron(III) complex, Polyhedron 20, 669 (2001).

[65] F. E. Mabbs, V. N. McLachlan, D. McFadden, and A. T. McPhail, Magnetic properties and crystal and molecular structure of $\mu$-oxo-bis[bis-(2-methyl-8-hydroxyquinolinato)iron(III)]chloroform, J. Chem. Soc., Dalton Trans., 2016 (1973).

[66] A. Jozwiuk, A. L. Ingram, D. R. Powell, B. Moubaraki, N. F. Chilton, K. S. Murray, and R. P. Houser, Redox and acid-base properties of asymmetric non-heme (hydr)oxo-bridged diiron complexes, Dalton Trans. 43, 9740 (2014).

[67] J. W. Shin, S. R. Rowthu, J. E. Lee, H. I. Lee, and K. S. Min, Syntheses, structures and magnetic properties of dinuclear oxo-bridged iron(III) complexes, Polyhedron 33, 25 (2012).

[68] R. E. Norman, R. C. Holz, S. Menage, L. Que, J. J. Zhang, and C. J. O'Connor, Structures and properties of dibridged ( $\mu$-oxo)diiron(III) complexes. Effects of the Fe-O-Fe angle, Inorg. Chem. 29, 4629 (1990).

[69] J. R. Hartman, R. L. Rardin, P. Chaudhuri, K. Pohl, K. Wieghardt, B. Nuber, J. Weiss, G. C. Papaefthymiou, R. B. Frankel, and S. J. Lippard, Synthesis and characterization of ( $\mu$-hydroxo)bis( $\mu$-acetato)diiron(II) and ( $\mu$-oxo)bis( $\mu$-acetato)diiron(III) 1,4,7-trimethyl1,4,7-triazacyclononane complexes as models for binuclear iron centers in biology; properties of the mixed valence diiron(II,III) species, J. Am. Chem. Soc. 109, 7387 (1987).

[70] W. H. Armstrong, A. Spool, G. C. Papaefthymiou, R. B. Frankel, and S. J. Lippard, Assembly and characterization of an accurate model for the diiron center in hemerythrin, J. Am. Chem. Soc. 106, 3653 (1984).

[71] W. Ghattas, Z. Serhan, N. E. Bakkali-Taheri, M. Réglier, M. Kodera, Y. Hitomi, and A. J. Simaan, Synthesis and characterization of a binuclear iron(III) complex bridged by 1-aminocyclopropane-1-carboxylic acid. Ethylene production in the presence of hydrogen peroxide, Inorg. Chem. 48, 3910 (2009). 
[72] J. B. Vincent, J. C. Huffman, G. Christou, Q. Li, M. A. Nanny, D. N. Hendrickson, R. H. Fong, and R. H. Fish, Modeling the dinuclear sites of iron biomolecules: synthesis and properties of $\mathrm{Fe}_{2} \mathrm{O}(\mathrm{OAc})_{2} \mathrm{Cl}_{2}$ (bipy) $)_{2}$ and its use as an alkane activation catalyst, J. Am. Chem. Soc. 110, 6898 (1988).

[73] A. Cornia, L. Gregoli, C. Danieli, A. Caneschi, R. Sessoli, L. Sorace, A.-L. Barra, and W. Wernsdorfer, Slow quantum relaxation in a tetrairon(III) single-molecule magnet, Inorg. Chim. Acta 361, 3481 (2008).

[74] C. Schlegel, E. Burzurí, F. Luis, F. Moro, M. Manoli, E. K. Brechin, M. Murrie, and J. van Slageren, Magnetic properties of two new $\mathrm{Fe}_{4}$ single-molecule magnets in the solid state and in frozen solution, Chem. Eur. J. 16, 10178 (2010).

[75] J. K. McCusker, J. B. Vincent, E. A. Schmitt, M. L. Mino, K. Shin, D. K. Coggin, P. M. Hagen, J. C. Huffman, G. Christou, and D. N. Hendrickson, Molecular spin frustration in the $\left[\mathrm{Fe}_{4} \mathrm{O}_{2}\right]^{8+}$ core: synthesis, structure, and magnetochemistry of tetranuclear iron-oxo complex $\left[\mathrm{Fe}_{4} \mathrm{O}_{2}\left(\mathrm{O}_{2} \mathrm{CR}\right)_{7}(\mathrm{bpy})_{2}\right]\left(\mathrm{C} 1 O_{4}\right)(\mathrm{R}=\mathrm{Me}, \mathrm{Ph})$, J. Am. Chem. Soc. 113, 3012 (1991).

[76] P. Chaudhuri, M. Winter, P. Fleischhauer, W. Haase, U. Flörke, and H. J. Haupt, Synthesis, structure and magnetism of a tetranuclear Fe(III) complex containing an $\left[\mathrm{Fe}_{4}\left(\mu_{3}-\mathrm{O}\right)_{2}\right]^{8+}$ core, Inorg. Chim. Acta 212, 241 (1993).

[77] E. Epifanovsky, T. B. Gilbert, X. Feng, J. Lee, Y. Mao, N. Mardirossian, P. Pokhilko, A. F. White, M. P. Coons, A. L. Dempwolff, Z. Gan, D. Hait, P. R. Horn, L. D. Jacobson, I. Kaliman, J. Kussmann, A. W. Lange, K. U. Lao, D. S. Levine, J. Liu, S. C. McKenzie, A. F. Morrison, K. D. Nanda, F. Plasser, D. R. Rehn, M. L. Vidal, Z.-Q. You, Y. Zhu, B. Alam, B. J. Albrecht, A. Aldossary, E. Alguire, J. H. Andersen, V. Athavale, D. Barton, K. Begam, A. Behn, N. Bellonzi, Y. A. Bernard, E. J. Berquist, H. G. A. Burton, A. Carreras, K. CarterFenk, R. Chakraborty, A. D. Chien, K. D. Closser, V. Cofer-Shabica, S. Dasgupta, M. de Wergifosse, J. Deng, M. Diedenhofen, H. Do, S. Ehlert, P.-T. Fang, S. Fatehi, Q. Feng, T. Friedhoff, J. Gayvert, Q. Ge, G. Gidofalvi, M. Goldey, J. Gomes, C. E. González-Espinoza, S. Gulania, A. O. Gunina, M. W. D. Hanson-Heine, P. H. P. Harbach, A. Hauser, M. F. Herbst, M. Hernández Vera, M. Hodecker, Z. C. Holden, S. Houck, X. Huang, K. Hui, B. C. Huynh, M. Ivanov, A. Jász, H. Ji, H. Jiang, B. Kaduk, S. Kähler, K. Khistyaev, J. Kim, G. Kis, P. Klunzinger, Z. Koczor-Benda, J. H. Koh, D. Kosenkov, L. Koulias, T. Kowalczyk, C. M. Krauter, K. Kue, A. Kunitsa, T. Kus, I. Ladjánszki, A. Landau, K. V. Lawler, D. Lefrancois, 
S. Lehtola, R. R. Li, Y.-P. Li, J. Liang, M. Liebenthal, H.-H. Lin, Y.-S. Lin, F. Liu, K.-Y. Liu, M. Loipersberger, A. Luenser, A. Manjanath, P. Manohar, E. Mansoor, S. F. Manzer, S.-P. Mao, A. V. Marenich, T. Markovich, S. Mason, S. A. Maurer, P. F. McLaughlin, M. F. S. J. Menger, J.-M. Mewes, S. A. Mewes, P. Morgante, J. W. Mullinax, K. J. Oosterbaan, G. Paran, A. C. Paul, S. K. Paul, F. Pavošević, Z. Pei, S. Prager, E. I. Proynov, A. Rák, E. RamosCordoba, B. Rana, A. E. Rask, A. Rettig, R. M. Richard, F. Rob, E. Rossomme, T. Scheele, M. Scheurer, M. Schneider, N. Sergueev, S. M. Sharada, W. Skomorowski, D. W. Small, C. J. Stein, Y.-C. Su, E. J. Sundstrom, Z. Tao, J. Thirman, G. J. Tornai, T. Tsuchimochi, N. M. Tubman, S. P. Veccham, O. Vydrov, J. Wenzel, J. Witte, A. Yamada, K. Yao, S. Yeganeh, S. R. Yost, A. Zech, I. Y. Zhang, X. Zhang, Y. Zhang, D. Zuev, A. Aspuru-Guzik, A. T. Bell, N. A. Besley, K. B. Bravaya, B. R. Brooks, D. Casanova, J.-D. Chai, S. Coriani, C. J. Cramer, G. Cserey, A. E. DePrince, R. A. DiStasio, A. Dreuw, B. D. Dunietz, T. R. Furlani, W. A. Goddard, S. Hammes-Schiffer, T. Head-Gordon, W. J. Hehre, C.-P. Hsu, T.-C. Jagau, Y. Jung, A. Klamt, J. Kong, D. S. Lambrecht, W. Liang, N. J. Mayhall, C. W. McCurdy, J. B. Neaton, C. Ochsenfeld, J. A. Parkhill, R. Peverati, V. A. Rassolov, Y. Shao, L. V. Slipchenko, T. Stauch, R. P. Steele, J. E. Subotnik, A. J. W. Thom, A. Tkatchenko, D. G. Truhlar, T. Van Voorhis, T. A. Wesolowski, K. B. Whaley, H. L. Woodcock, P. M. Zimmerman, S. Faraji, P. M. W. Gill, M. Head-Gordon, J. M. Herbert, and A. I. Krylov, Software for the frontiers of quantum chemistry: An overview of developments in the Q-Chem 5 package, J. Chem. Phys. 155, 084801 (2021).

[78] A. I. Krylov and P. M. W. Gill, Q-Chem: An engine for innovation, WIREs: Comput. Mol. Sci. 3, 317 (2013).

[79] A.D. Becke, Density-functional thermochemistry. III. The role of exact exchange, J. Chem. Phys. 98, 5648 (1993).

[80] C. Adamo and V. Barone, Toward reliable density functional methods without adjustable parameters: The PBE0 model, J. Chem. Phys. 110, 6158 (1999).

[81] Y. Zhao and D.G. Truhlar, The M06 suite of density functionals for main group thermochemistry, thermochemical kinetics, noncovalent interactions, excited states, and transition elements: two new functionals and systematic testing of four M06-class functionals and 12 other functionals, Theor. Chem. Acc. 120, 215 (2008).

[82] T. Yanai, D.P. Tew, and N.C. Handy, A new hybrid exchange-correlation functional using 
the Coulomb-attenuating method (CAM-B3LYP), Chem. Phys. Lett. 393, 51 (2004).

[83] M. A. Rohrdanz and J. M. Herbert, Simultaneous benchmarking of ground-and excited-state properties with long-range-corrected density functional theory, J. Chem. Phys. 129, 034107 (2008).

[84] M.A. Rohrdanz, K.M. Martins, and J.M. Herbert, A long-range-corrected density functional that performs well for both ground-state properties and time-dependent density functional theory excitation energies, including charge-transfer excited states, J. Chem. Phys. 130, $054112(2009)$.

[85] J.-D. Chai and M. Head-Gordon, Long-range corrected hybrid density functionals with damped atom-atom dispersion interactions, Phys. Chem. Chem. Phys. 10, 6615 (2008).

[86] N. Mardirossian and M. Head-Gordon, $\omega B 97 M-V:$ A combinatorially optimized, rangeseparated hybrid, meta-GGA density functional with VV10 nonlocal correlation, J. Chem. Phys. 144, 214110 (2016).

[87] G. Rajaraman, E. Ruiz, J. Cano, and S. Alvarez, Theoretical determination of the exchange coupling constants of a single-molecule magnet $\mathrm{Fe}_{10}$ complex, Chem. Phys. Lett. 415, 6 (2005).

[88] C. Benelli, S. Parsons, G. A. Solan, and R. E. P. Winpenny, Ferric wheels and cages: Decanuclear iron complexes with carboxylato and pyridonato ligands, Angew. Chem., Int. Ed. 35, 1825 (1996).

[89] A.-A. H. Abu-Nawwas, J. Cano, P. Christian, T. Mallah, G. Rajaraman, S. J. Teat, R. E. P. Winpenny, and Y. Yukawa, An Fe(III) wheel with a zwitterionic ligand: the structure and magnetic properties of $\left[\mathrm{Fe}(\mathrm{OMe})_{2} \text { (proline) }\right]_{12}\left[\mathrm{ClO}_{4}\right]_{12}$, Chem. Comm. , 314 (2004). 